\title{
Dissecting the Potential Roles of Nigella sativa and Its Constituent Thymoquinone on the Prevention and on the Progression of Alzheimer's Disease
}

\author{
Marco Cascella ${ }^{1 \dagger}$, Sabrina Bimonte ${ }^{1 * \dagger}$, Antonio Barbieri ${ }^{2}$, Vitale Del Vecchio ${ }^{2}$, \\ Maria Rosaria Muzio ${ }^{3}$, Andrea Vitale ${ }^{4}$, Giulio Benincasa ${ }^{4}$, Anna Bella Ferriello ${ }^{4}$, \\ Amalia Azzariti ${ }^{5}$, Claudio Arra ${ }^{2}$ and Arturo Cuomo ${ }^{1}$ \\ ${ }^{1}$ Division of Anesthesia and Pain Medicine, Istituto Nazionale Tumori, IRCCS Fondazione G. Pascale, Naples, Italy, ${ }^{2}$ S.S.D. \\ Sperimentazione Animale, Istituto Nazionale Tumori, IRCCS Fondazione G. Pascale, Naples, Italy, ${ }^{3}$ Division of Infantile \\ Neuropsychiatry, UOMI-Maternal and Infant Health, Naples, Italy, ${ }^{4}$ Pineta Grande Hospital, Caserta, Italy, ${ }^{5}$ Experimental \\ Pharmacology Laboratory, IRCCS Istituto Tumori Giovanni Paolo II, Bari, Italy
}

Several nutraceuticals have been investigated for preventing or retarding the progression of different neurodegenerative diseases, including Alzheimer's disease (AD). Because Nigella sativa (NS) and its isolated compound thymoquinone (TQ) have significant anti-oxidant and anti-inflammatory proprieties, they could represent effective neuroprotective agents. The purpose of this manuscript is to analyze and to recapitulate the results of in vitro and in vivo studies on the potential role of NS/TQ in AD's prevention and treatment. The level of evidence for each included animal study has been assessed by using a modified CAMARADES (Collaborative Approach to Meta-Analysis and Review of Animal Data from Experimental Studies) 10-item checklist. We used MEDLINE and EMBASE databases to screen relevant articles published up to July 2017. A manual search was also performed. The database search yielded 38 studies, of which 18 were included in this manuscript. Results from these approaches suggest that NS or TQ could represent an effective strategy against $A D$ due to the balancing of oxidative processes and the binding to specific intracellular targets. The overall effects mainly regard the prevention of hippocampal pyramidal cell loss and the increased cognitive functions.

Keywords: Nigella sativa, thymoquinone, natural compounds, Alzheimer's disease, oxidative stress

\section{INTRODUCTION}

Although the knowledge of the exact pathophysiological mechanisms remains an unresolved issue, an emerging evidence underlines the role of the oxidative damage and microglia-mediated neuro-inflammatory responses in the initiation of neurodegenerative disorders including Alzheimer's disease (AD) (Mosher and Wyss-Coray, 2014). Experimental findings demonstrated that in $\mathrm{AD}$ pathogenesis, the $4.2-\mathrm{kD}$ amyloid $\beta$ peptide $(\mathrm{A} \beta)$-dependent microglial activation leads to neuronal injury through a complex cascade by involving the secretion of various pro-inflammatory molecules such as tumor necrosis factor- $\alpha$ (TNF- $\alpha$ ), interleukin (IL) IL-6, IL-1 $\beta$, reactive oxygen species (ROS), and reactive nitrogen species (NOS) (Agostinho et al., 2010). In turn, the neuroinflammation and oxidative stress processes are responsible for the impairment of 
the neurovascular working leading to an axonal demyelination, local hypoxia-ischemia, and to restoring white matter damages (Iadecola, 2010).

Differently from a big amount of data relating to its pathogenesis, a poor number of $\mathrm{AD}$ therapeutics has been developed so far. Thus, many investigations have been performed to evaluate new neuroprotective agents. For instance, several clinical studies suggested that a high dietary antioxidant intake (e.g., vitamin E, flavonoids, and omega-3 fatty acids) could reduce the risk of $\mathrm{AD}$ (Andrade and Radhakrishnan, 2009). On the contrary, it has been reported that high dosage of B vitamin supplements (folate, B6, and B12) was not able to retard cognitive decline in patients with mild to moderate AD (Aisen et al., 2008). Moreover, in addition to the clinical investigations most experimental studies have been conducted in vitro or in animal models of $\mathrm{AD}$ to test natural compounds with antioxidants proprieties such as epigallocatechin gallate (EGCG) (Cascella et al., 2017a), quercetin (Schültke et al., 2003), kaempferol (Lei et al., 2012), resveratrol (Zhao et al., 2015) as potential protective factors to neurodegeneration induced by oxidative stress (Mecocci et al., 2014; Essa et al., 2016; De Domenico and Giudetti, 2017).

Furthermore, plant compounds and derivates could represent an important strategy for preventing or delaying the functional decline in AD's patients (Laver et al., 2016). For instance, Ginkgo biloba which shows rapid penetration and absorption into the brain is a well-studied tree in AD (Yuan et al., 2017), whereas curcumin reduced $\mathrm{A} \beta$-related cerebral burden and neuroinflammation in transgenic AD mice (Lim et al., 2001). Thus, there is a great interest about the nutrient's role in the development of new preventive and therapeutic areas regarding $\mathrm{AD}$ and other neurodegenerative disorders (Poulose et al., 2017).

Nigella sativa (NS), also identified as black seed or black cumin, is a flowering plant belonging to the Family

\footnotetext{
Abbreviations: $\mathrm{AD}$, Alzheimer's disease; $\mathrm{A} \beta$, amyloid $\beta$ peptide; TNF- $\alpha$, tumor necrosis factor- $\alpha$; IL, interleukin; ROS, reactive oxygen species; NOS, reactive nitrogen species; EGCG, epigallocatechin gallate; NS, Nigella sativa; PUFA, polyunsaturated fatty acids; TQ, thymoquinone; PRISMA, Preferred Reporting Items for Systematic Reviews and Meta-Analyses; CAMARADES, Collaborative Approach to Meta-Analysis and Review of Animal Data from Experimental Studies; CGNs, cerebellar granule neurons; AChE, acetylcholinesterase; NOS, Nigella sativa oil; HF, hexane fraction; EAF, ethyl acetate fraction; WF, water fraction; LDH, lactate dehydrogenase; iNOS, inducible nitric oxide synthase; MMPs, matrix metalloproteinases; hiPSC, human induced pluripotent stem cells; $\alpha \mathrm{SN}, \alpha$-synuclein; FM1-43 dye, $N$-(3-Triethylammoniumpropyl)-4-(4(Dibutylamino) Styryl) Pyridinium Dibromide; IFN $\gamma$, Interferon-gamma; Ccl5, chemokine (C-C) motif ligand; Nos2, nitric oxide synthase 2 inducible; Ptgs2, pros-taglandin-endoperoxide synthase 2; Txnip, thioredoxin-interacting protein; Peroxiredoxin 1, Prdx 1; Sxrn1, sulfiredoxin 1 homolog (S. cerevisiae); PGLA, Poly Lactic-co-Glycolic Acid; pDNA, plasmid DNA; 2VO, bilateral carotid arteries occlusion; IP, intraperitoneal; IIP, intraippocampal; IRI, ischemia-reperfusion injury; TBRAS, thiobarbituric acid reactive substances; MDA, malondialdehyde; LPS, lipopolysaccharide; PAM, positive allosteric modulator; MT, metformin; GI, glimepiride; nAChR, nicotin acetyl choline receptor; MSCs, mesenchymal stem cells; STZ-T2DM, streptozotocin-induced diabetes; XO, xanthine oxidase; GPx, glutathione peroxidase; SOD, superoxide dismutase; GST, glutathione Stransferase; AGEs, advanced glycation end products; M1, microglia phenotype 1; M2, microglia phenotype 2; NF-kB, nuclear factor kappa-light-chain-enhancer of activated B cells.
}

Ranunculaceae. In several countries such as the Middle East, Northern Africa and Southwest Asia, NS's seeds and its derivative, have been used not only as a spice and food preservative, but also as a protective and curative remedy for many disorders (Gholamnezhad et al., 2016; Hayatdavoudi et al., 2016). Chemically, NS contains $30 \%$ fixed oils (mainly fatty acids), $0.40-0.45 \%$ volatile oils, vitamins, amino acids, proteins, carbohydrates, alkaloids, saponins, crude fiber, and minerals. NS oil (NSO) is rich in polyunsaturated fatty acids (PUFA), phytosterols, TQ (up to $25 \%$ in volatile oil), carvacrol, t-anethole, sesquiterpenelongifolene, and 4 terpinol (Liu et al., 2011; Akram Khan and Afzal, 2016).

It is of note that TQ has anti-oxidant and anti-inflammatory proprieties (Ragheb et al., 2009). This compound, indeed, has an effect on free radical scavenging enhanced by many factors, such as its redox features and its ability to bypass the biological barriers and to move into subcellular compartments (Badary et al., 2003). Consequently, several investigations showed the antiasthmatic (Keyhanmanesh et al., 2010), antibacterial (Boudiaf et al., 2016), antidiabetic (Bamosa et al., 2010), hepatoprotective (Yildiz et al., 2008), antinociceptive (Abdel-Fattah et al., 2000), and antihypertensive (Jaarin et al., 2015) properties of NS and TQ. Furthermore, other researchers focused the studies on their anticancer activities (Kundu et al., 2014) as well as on their potential role in the prevention and/or treatment of toxic effects of anticancer drugs (e.g., chemotherapy-induced nephrotoxicity) (Cascella et al., 2017b). Important findings suggest that TQ can be considered an additive treatment option in neurological and neurosurgical practice (Elmaci and Altinoz, 2016). Detailed description on the protective roles of $N$. sativa and TQ in multiple disorders have been also described by Javidi et al. (2016), and Sahak et al. (2016). Moreover, the authors identified in N. sativa a potential candidate for the treatment of physiological and neurological disorders.

Recently, Bin Sayeed et al. (2013) showed that NS (500 mg capsule twice daily for 9 weeks) enhanced memory, attention, and cognition in healthy human volunteers. In another paper, the authors found that a reduced assumption of NS (500 mg once daily for 4 weeks) was enough to stabilize mood, decrease anxiety and modulate cognition positively in healthy adolescent males, compared to placebo (Bin Sayeed et al., 2014). The beneficial effects of NSO on learning and memory abilities were also confirmed in healthy male adult Sprague Dawley rats (Sahak et al., 2013).

In a light of these findings, our purpose is to analyze and to recapitulate the outcome of the pre-clinical studies on the potential roles of NS, and its constituent TQ, for preventing and slowing the AD's progression.

\section{BIBLIOGRAFICH RESEARCH}

This review was written according to Preferred Reporting Items for Systematic Reviews and Meta-Analyses (PRISMA) guidelines (Liberati et al., 2009). An implementation to the research was obtained by computer-operated search strategy using Medline and Embase databases up to July 2017. Two reviewers (M.C. and S.B.) engineered the review protocol, 
selected the inclusion and exclusion criteria and assessed the potential articles for the inclusion into the review. The search was performed by using the following terms to detect the specific nutraceutical ("thymoquinone" OR "Nigella sativa") combined with the clinical condition ("Alzheimer's" OR "dementia") or the term ("memory"). Additionally, was performed a manual search of the reference lists of identified articles. Inclusion criteria for study selection were as follows: performed in cell cultures, on animals, systematic reviews and meta-analysis on the topic. All genders and strains were included for animal studies. Published abstracts, summarizing studies, were excluded. Two independent reviewers (V.D.V. and A.B.) screened and assessed for inclusion, the articles identified by the search. The two reviewers agreed on $95 \%$ of the selected article and reached consensus on all included studies through collective discussions with a third reviewer (M.R.M.).

\section{RESULTS}

Firstly, the search strategy (showed in Figure 1) identified 31 articles. Thanks to the manual search, additional 7 records were identified and 15 duplicated were removed. Consequently, 23 non-duplicated articles were identified and full texts recovered. After the record screening, 4 articles were excluded ( 1 full text not available and 3 review articles). Of the remaining 19, 1 study was excluded for the reasons reported in the flow chart. Thus, 16 eligible studies were included in this review. For the narrative synthesis, we divided the available studies into two categories: in vitro studies: an overview on the molecular mechanism underlying the anti-Alzheimer effects of NS or TQ, and Pre-clinical in vivo studies on the preventive effects of NS or TQ on $A D$. The quality of evidence of each pre-clinical in vivo study was assessed by using a modified CAMARADES (Collaborative Approach to Meta-Analysis and Review of Animal Data from Experimental Studies) 10-item checklist (Macleod et al., 2004). Two authors (A.B.F. and A.V.) independently assessed the study's quality and any disagreements were solved through discussion or consultation with the corresponding author.

\section{In Vitro Studies: An Overview on the Molecular Mechanism Underlying the Anti-Alzheimer Effects of NS or TQ}

Several in vitro studies on the anti-oxidant and antineuroinflammatory properties of TQ have been conducted on different cells lines, including primary rat cerebellar granule neurons (CGNs), PC-12, E18, hi-PSC, SH-Y5Y, BV-2, and N2a cells as showed in Table 1. Evidences from these studies, strongly suggested that TQ has a great potential against neurotoxicity induced by $A ß$ peptide which, in turn, plays a pivotal role in AD's pathogenesis.
Records identified by database searching $(\mathrm{n}=31)$
Additional records identified by other sources

$$
(n=7)
$$

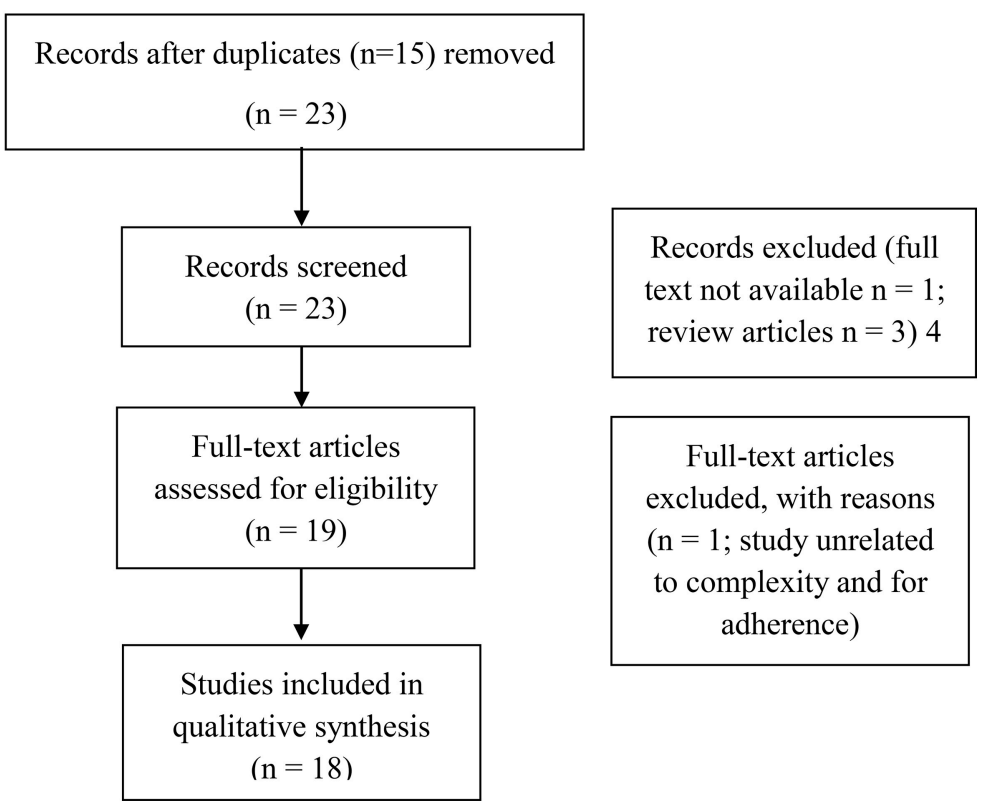

FIGURE 1 | PRISMA flow diagram. 
TABLE 1 | In vitro studies on the effects of NS or TQ in AD.

\begin{tabular}{|c|c|c|c|}
\hline Cell lines/in vitro test & Drugs and dosages & Main results & References \\
\hline $\begin{array}{l}\text { Dried plant /AChE inhibition } \\
\text { assay }\end{array}$ & $\begin{array}{l}\text { Galanthamine; Thymohydro-quinone } \\
\text { Carvacrol; Thymol; Linalool. (10-0,00001 } \\
\text { mg/mL) }\end{array}$ & $\begin{array}{l}\text { AChE inhibitory potential decreased as follows: Galanthamine } \\
>\text { Thymohydro-quinone }>\text { Carvacrol }>\mathrm{TQ}>\text { Total essential } \\
\text { oil }>\text { Thymol }>\text { Linalool. }\end{array}$ & Jukic et al., 2007 \\
\hline Primary rat CGNs & NSO (500-2 mg/mL) & $\begin{array}{l}\text { NSO and its fractions prevented the A } \beta \text { toxicity (NSO, and WF } \\
\text { more than HF, and EAFs). }\end{array}$ & Ismail et al., 2008 \\
\hline PC-12 & TQ $(0.78-400 \mu \mathrm{M})$ & $\begin{array}{l}\text { TQ: (1) ameliorated induced loss of cell viability; (2) prevented } \\
\text { the AB25-35-induced increase activity of LDH; (3) had } \\
\text { protective effects on GPx, GR, and AChE in PC } 12 \text { cells } \\
\text { exposed to AB25-35; (4) downregulated the iNOS expression } \\
\text { along with NO level; (5) had a protective role of intracellular } \\
\text { oxidative stress, by restoring the ROS level; (6) augmented the } \\
\text { membrane potential by restoring the normal level of MMPs. }\end{array}$ & Khan et al., 2012 \\
\hline E18 & TQ $(0.1,1,10,100 \mathrm{nM})$ & $\begin{array}{l}\text { TQ: (1) reduced intracellular ROS level in neurons treated with } \\
A ß 1-42 ;(2) \text { reduced } A ß \text {-induced inhibition of synaptic vesicle } \\
\text { recycling. }\end{array}$ & Alhebshi et al., 2013 \\
\hline CGNs & $\mathrm{TQ}(0.1$ and $1 \mu \mathrm{M})$ & $\begin{array}{l}\text { TQ had protective effects on } A B 1-40 \text {-induced neurotoxicity } \\
\text { by reducing the production of free radical on } A \beta 1-40 \text { in } C G N s \\
\text { and by attenuating the activation of Caspase- } 3,-8 \text {, and- } 9 \\
\text { on } A B 1-40 \text { exposure. }\end{array}$ & Ismail et al., 2013 \\
\hline $\begin{array}{l}\text { Rat primary hippocampal cells; } \\
\text { hiPSC-derived neurons }\end{array}$ & TQ (100 nM) & $\begin{array}{l}\text { TQ: (1) neurons were protected against SN-induced synapse } \\
\text { damage and the synaptophysin was enhanced; (2) } \\
\text { maintained the synaptic activities in hippocampal neurons } \\
\text { were maintained; (3) the uptake of FM1-43 dye increased, } \\
\text { while the inhibitory effect of SN on synaptic vesicle recycling } \\
\text { was decreased. }\end{array}$ & Alhebshi et al., 2014 \\
\hline SH-SY5Y & TQ, EGCG; DLPC & $\begin{array}{l}\text { TQ prevented the oxidation of } \mathrm{A} B \text { by decreasing the } \\
\text { expression of } \mathrm{NO} \text { and by increasing the GSH levels. }\end{array}$ & Kennedy et al., 2014 \\
\hline BV-2 & $\mathrm{TQ}(0-40 \mu \mathrm{M})$ & $\begin{array}{l}\text { TQ treatment in the LPS/IFN } \gamma \text {-activated microglia altered the } \\
\text { expression profiles of Ccl5, Nos and Ptgs } 2 \text {. }\end{array}$ & $\begin{array}{l}\text { Cobourne-Duval et al., } \\
2016\end{array}$ \\
\hline $\mathrm{N} 2 \mathrm{a}$ & $\begin{array}{l}\text { NOS encapsulated in Nanoparticles-pDNA } \\
\text { (ratio from } 5 \text { to } 50 \% \text { ) }\end{array}$ & Encapsulated NSO promoted neurite outgrowth of N2a cells. & Doolaanea et al., 2016 \\
\hline
\end{tabular}

AChE, acetylcholinesterase; NSO, Nigella Sativa Oil; TQ, Thymoquinone; CGNs, cerebellar granule neurons; HF, hexane fraction; EAF, ethyl acetate fraction; WF, water fraction; GPX, glutathione peroxidase; GR, glutathione reductase; AB, amyloid beta; iNOS, Inducible nitric oxide synthase; NOS, nitric oxide synthase; ROS, Reactive oxygen species; MMPs, Matrix metalloproteinases; B-amyloid peptide 1-40 sequence, A 1-4; human induced pluripotent stem cells (hiPSC); $\alpha$-synuclein, $\alpha S N$; (FM1-43 dye, N-(3-Triethylammoniumpropyl)-4-(4-

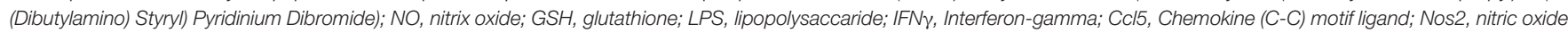
synthase 2 inducible; Ptgs2, Pros-taglandin-endoperoxide synthase 2; Txnip, Thioredoxin-interacting protein; Prdx 1, Peroxiredoxin 1; S. cerevisiae, Sulfiredoxin 1 homolog.

Jukic et al. (2007) reported that the principal constituents of essential oil Thymus vulgaris, thymol and carvacrol, and their derivatives (e.g., TQ and thymohydroquinone) had inhibitory effects on acetylcholinesterase (AChE) thanks to their antioxidant properties. Thus, indicating that these compounds can be identified as potential therapeutic options for the treatment of $\mathrm{AD}$ and/or of cognitive disorders. Ismail et al. (2008) reported findings on the neuroprotective role of NSO and its fractions [(e.g., hexane fraction (HF), ethyl acetate fraction (EAF) and water fraction $(\mathrm{WF})$ ] against $\mathrm{A} B$-induced cell death in primary rat CGNs. In these in vitro investigations, was reported that WF and NSO had a better protective effect on $A \beta$-induced toxicity in CGNs than HF and EAFs fractions, by acting on the antioxidant pathway. Khan et al. (2012), showed that TQ was able to reduce the neurotoxicity induced by $A B$ in an in vitro model of undifferentiated pheochromocytoma rat cell line, PC-12, by inhibiting the oxidative stress and the mitochondrial dysfunction through the reestablishment of the abnormal levels of Matrix metalloproteinases (MMPs) and ROS. These data confirmed the hypothesis that $\mathrm{TQ}$, though the mechanism of neuroprotection, may be potentially used in the management of neurodegenerative disorders, including AD. Similar findings were obtained by a study conducted by Alhebshi et al. (2013). The authors proved that TQ played a role in the neuroprotection against neurotoxicity induced by $A ß$ in rat primary neurons, E18, through the inhibition of ROS formation, and mitochondrial membrane depolarization. Moreover, they showed that TQ reduced $A ß$-induced inhibition of synaptic vesicle recycling. The neuroprotective properties of TQ in $\mathrm{AD}$ were also confirmed by a study conducted on CGNs cells exposed $A \beta 1-40$ sequence (A $\beta 1-40)$ neurotoxicity (Ismail et al., 2013). Authors showed that the pre-treatment with TQ $(0.1$ and $1 \mu \mathrm{M})$ was able to prevent the generation of free radicals, and the apoptosis via caspase pathways, thus resulting in an improvement of cell viability. Additionally, TQ preserved the extensive neurite network and the morphology of cell bodies on Ab1-40-induced morphological damages in CGNs. Subsequently, Alhebshi et al. described a neuroprotective role of TQ and its potential application in patients with Parkinson's disease and dementia with Lewy bodies. Specifically, it has been showed that TQ protected cultured rat 
primary hippocampal and human induced pluripotent stem cells (hiPSC)-derived neurons against $\alpha$-synuclein $(\alpha S N)$ induced synaptic toxicity, by acting on synaptic vesicle recycling (Alhebshi et al., 2014). Interesting findings on the role of TQ as a potential preventive agent for AD's treatment, were also reported for human neuroblastoma cells, SH-SY5Y by Kennedy et al. (2014). To this regard, the authors demonstrated that TQ and other antioxidants [e.g., EGCG, dilinoleoylphosphatidylcholine (DLPC)] were able to prevent oxidation of Aß, by acting on TNF$\alpha$-mediated signaling pathway, through the downregulation of nitric oxide (NO) and the upregulation of glutathione (GSH). Recently (Cobourne-Duval et al., 2016), it has been demonstrated that TQ had antioxidant effects in activated BV-2 murine microglial cells, by acting on ROS and proinflammatory cytokines levels whose expression are extremely higher in several neurodegenerative disorders, including AD. Basically, the authors showed that the treatment with TQ of the lipopolisaccaride (LPS)/Interferon-gamma (IFN $\gamma$ ) activated microglia, impaired the expression profiles of specific genes involved in the oxidation process. Specifically, TQ was able to downregulate the expression of Chemokine (C-C) motif ligand 5 (Ccl5), nitric oxide synthase 2, inducible (Nos2), Prostaglandin-endoperoxide synthase 2 (Ptgs2), Thioredoxininteracting protein (Txnip) and to upregulate the expression of Peroxiredoxin 1 (Prdx 1) and Sulfiredoxin 1 homolog (S. cerevisiae) (Sxrn1). Interestingly, by using an innovative technology based on the application of Poly Lactic-co-Glycolic Acid (PLGA) nanoparticles, Doolaanea et al. co-encapsulated NSO and plasmid DNA (pDNA) with PLGA nanoparticles in order to enhance gene therapy of AD's syndrome. The authors demonstrated that the encapsulated NSO promoted the outgrowth of murine neuroblastoma cells BV-2, thus resulting in a better efficacy for AD's treatment (Doolaanea et al., 2016).

Taken together, these findings strongly suggest that NS, or its constituent TQ, could represent an effective therapeutic agent with neuroprotective properties against $\mathrm{AD}$, due to the action on different molecular pathways involved in its pathogenesis. Despite these encouraging results, more studies will be needed to translate these pieces of knowledge into clinical practice.

\section{Pre-clinical in Vivo Studies on Preventive Effects of NS or TQ on AD}

The neuroprotective effects of NS and/or its constituent TQ have been also demonstrated by in vivo experiments as summarized in Table 2. Several experiments were performed on rat models of global cerebrovascular hypo perfusion, leading to hippocampus neurodegeneration and cognitive defects. The first study was reported by Al-Majed et al. (2006) in a rat model of transient forebrain ischemia-induced neural damage. Results from this study showed that TQ pretreatment significantly reduced the loss of hippocampal neuronal cells $(P<0.001)$, in the CA1 region. This effect was correlated with antioxidant activities of TQ on the levels of malondialdehyde, GSH, catalase, and on the superoxide dismutase (SOD) activities. A similar rat model was used by Hosseinzadeh et al. (2007). It was demonstrated that TQ and NSO had significant neuroprotective effect on hippocampal cells of rats, mainly correlated with the inhibition of lipid peroxidation after cerebral ischemia.
More recently, Hobbenaghi et al. (2014) by administrating NS in rats during the carotid clamp, showed that the strongest neuroprotective effect of NS, identified as the prevention of intracellular edema of hippocampal interneurons and astrocytes, was obtained with the highest dose $(50 \mathrm{mg} / \mathrm{kg})$. Later on, Azzubaidi et al. (2012) conducted behavioral investigations of animals by using the two-vessel occlusion (2VO) murine model. This model is obtained by a ligation and a cutting of the common carotid arteries, thus representing an optimal strategy to prove the so-called vascular hypothesis of $\mathrm{AD}$ responsible for neurodegeneration. In these experiments, long-term memory, short-term memory and working memory were investigated through the Morris water maze (MWM). Results showed that the oral administration of NSO $(1 \mathrm{mg} / \mathrm{kg})$ for 10 days prior to $2 \mathrm{VO}$ surgery and then for 70 days post $2 \mathrm{VO}$ surgery, highly preserved the spatial cognitive functions with an overall memory's and learning's improvements.

Azizi et al. (2012) reported an interesting study on the efficacy of Thymol and cravacrol, monoterpenic phenols also present in NS, against cognitive deficits in rat model of AD. Results suggested that the reduction of cognitive defects induced by treatment with thymol and carvacrol, was probably associated to their antioxidant and anti-inflammatory properties. Moreover, a lower toxicity of both compounds was also reported.

Recently, in an animal model of AD [lipopolysaccharide(LPS)-induced neuroinflammation in rats] it was tested the combination of PNU-120596, a positive allosteric modulator (PAM) of $\alpha 7$ nicotine acetyl choline receptor (nAChR), with the specific nAChR agonist PNU282987 or with TQ (Ibrahim AbdEl Fattah et al., 2016). Data reported that TQ or the $\alpha 7 \mathrm{nAChR}$ agonist in combination with the PAM had a pivotal role in the treatment of $\mathrm{AD}$, by reducing the neuroinflammation, and by activating the mesenchymal stem cells (MSCs). Indeed, by molecular [antiphospho-cyclic adenosine phosphate (cAMP) response element binding protein (P-CREB), and CD44 Ab], and morphometric analyses (evaluation of the area of deformed neurons, and $A \beta$ plaques) were reported a reduced number of deformed neurons and glial cells as well as an increased number of pyramidal cells. Interestingly, the combination of TQ, or $\alpha 7 \mathrm{nAChR}$ agonist, with PAM had a better effect in comparison with TQ alone.

A possible role of the brain insulin resistance and a reduced insulin signaling in the AD's pathogenesis, was also described by several studies (Aliev et al., 2014). Baalba et al., by using streptozotocin-induced diabetic (STZ-T2DM) rats, showed that the daily intake of NSO induced the expression of insulin receptor and altered the expression of insulin-like growth factor-1 and phosphoinositide-3 kinase (Balbaa et al., 2016). Based on these results, subsequently, the authors showed that in STZ-T2DM rats, NSO $(2.0 \mathrm{ml} / \mathrm{Kg})$ and anti-diabetic drugs (metformin and glimepiride) alone and/or in combination, were able to suppress brain levels of oxidative stress markers such as xanthine oxidase (XO) and NOS, through the product of lipid peroxidation [e.g., thiobarbituric acid reactive substances (TBARS)] (Balbaa et al., 2017). On the contrary, an increased activity of antioxidant agents (e.g., glutathione) and enzymes such as glutathione peroxidase (GPx), SOD and glutathione S-transferase (GST), was observed. Furthermore, the NSO 
TABLE 2 | In vivo experiments on the anti-neurodegenerative properties of NS or TQ in AD.

\begin{tabular}{|c|c|c|}
\hline Animal models & Methods & Tests \\
\hline $\begin{array}{l}\text { Wister-albino rats with } \\
\text { ischemia-reperfusion injury } \\
(\mathrm{IRI}) \text { in hippocampus }\end{array}$ & $\begin{array}{l}\text { TQ ( } 5 \text { mg/kg/day p.o.) } 5 \text { days } \\
\text { before ischemia and continued } \\
\text { during the reperfusion time. }\end{array}$ & $\begin{array}{l}\text { Histological and Histochemic } \\
\text { studies }\end{array}$ \\
\hline
\end{tabular}

Sprague Dawley rats underwent to $2 \mathrm{VO}$ surgery

Wistar rats with $\mid \mathrm{RI}$

\author{
Wistar rats received IP \\ injection of $A \beta-25-35(1 \mu$ l \\ into the CA1 region) or \\ scopolamine (IP $1 \mathrm{mg} / \mathrm{kg}$ ) \\ Rats with experimentally \\ induced $A D$ (LPS)
}

Streptozotocin-Induced Diabetic Rats

\section{NSO ( $1 \mathrm{mg} / \mathrm{kg})$, OG daily for $10 \quad$ Memory tests days prior to $2 \mathrm{VO}$ surgery and then for 70 days post $2 \mathrm{VO}$} surgery.

NS (ip $1 \mathrm{mg} / \mathrm{kg}, 10 \mathrm{mg} / \mathrm{kg}$ and 50 $\mathrm{mg} / \mathrm{kg}$ ), during the carotid clamp and after $72 \mathrm{~h}$.

Thymol $(0.5,1$, or $2 \mathrm{mg} / \mathrm{kg})$; Carvacrol $(0.5,1$, or $2 \mathrm{mg} / \mathrm{kg})$ IP injected $30 \mathrm{~min}$ before MWM.

TQ (10 mg/kg IP), or nAChR agonist, plus PAM (for 5 days).

NSO (2 ml/Kg orally), MT (100 $\mathrm{mg} / \mathrm{Kg})$, Gl $(0.8 \mathrm{mg} / \mathrm{Kg})$, and the insulin receptor inhibitor IOMe-AG538 (21 days).
Rats with memory deficits induced by LPS.

Sprague Dawley rats fed with a high fat-cholesterol diet

\section{2, 5, or $10 \mathrm{mg} / \mathrm{kg}$ TQ extract $30 \mathrm{~min}$ before IP LPS}

TQ rich-fraction nanoemulsion (TQRFNE); TQ emulsion; TQ nanoemulsion.

\section{histopathological examinations}

Memory test; Acute toxicity

\section{Histological and Histochemical studies}

\begin{abstract}
Serum biochemical assays; Neuroinflammation cytokines profile; Brain oxidant and antioxidant markers expression Cholinergic function; AGEs and brain insulin resistance. Modification of brain AD-related miRNA expression profile, were observed
\end{abstract}

Behavioral tests (PA, MWM); Biochemical measurements in hippocampal and cortical tissues

Memory test (MWM); Serum antioxidant status; Genes expression levels in brain cortex and hippocampus

Results
TQ pre-treatment significantly
attenuated the loss of hippocampal
neuronal cells $(P<0.001)$, reduced
the malondialdehyde's level and
increased GSH, catalase and SOD
activities.
NSO had a protective effect on spatial
cognitive functions.

References

Al-Majed et al., 2006

Hosseinzadeh et al., 2007

\author{
Prevention of intracellular edema of \\ hippocampal interneurons and \\ Hobbenaghi et al., \\ 2014 \\ astrocytes with the highest dose (50 \\ $\mathrm{mg} / \mathrm{kg}$ ).
Thymol and carvacrol improved cognitive functions and reversed the \\ effect of $A \beta$ and scopolamine. \\ Azzubaidi et al., 2012
}
TQ, or a $\alpha 7 \mathrm{nAChR}$ agonist, in combination with PAM, attenuates neuroinflammation and activates MSCs.
NSO and the anti-diabetic drugs alone and/or in combination suppressed the oxidative stress, the amyloidogenic pathway and the pro-inflammatory mediator. A reduction in the insulin receptor inhibitory effect of IOMe-AG538 and a modification of the insulin-signaling pathway.
Azizi et al., 2012
Balbaa et al., 2016, 2017

TQ was able to enhance memory impairments by reducing the hippocampal cytokine levels and brain's damage.

TQRFNE ameliorated behavioral changes, lipid peroxidation and soluble A $\beta$ levels. Improved radical scavenging activity and increased antioxidants genes expression levels.
Bargi et al., 2017

Ismail et al., 2017

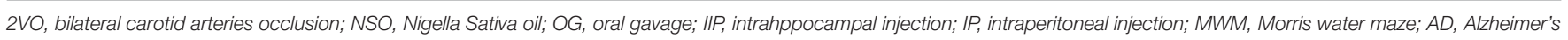

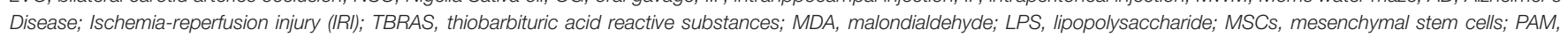

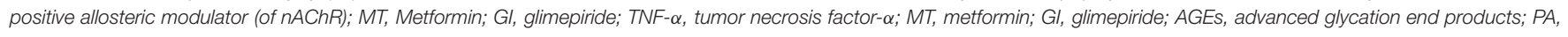
passive avoidance test; TQRFNE, TQ rich-fraction nanoemulsion.

administration lowered the insulin receptor inhibitory effect of IOMe-AG538 by modifying the insulin-signaling pathway and by reducing the levels of advanced glycation end products (AGEs) which are proteins or lipids involved in aging and in the pathogenesis of degenerative diseases, including AD. Again, the authors investigated on the modification of brain AD-related miRNA expression profile and demonstrated that NSO restored the brain tissue levels and serum values of miRNA, thus suggesting a possible role of miRNAs for the early diagnosis of $\mathrm{AD}$.

Bargi et al. (2017), reported that TQ was able, yet at lower doses, to improve memory impairments induced by LPS in rats. These behavioral results were associated with a reduction of the hippocampal neuroinflammatory cytokines TNF- $\alpha$, and IL6, a decreased level of markers of oxidative damage in brain tissues such as NOS, malondialdehyde (MDA) as well as an increased activity of SOD and catalase in hippocampus and cortex. Finally, in a recent paper Ismail et al. (2017) tested TQ rich-fraction nanoemulsion (TQRFNE) compared to TQ conventional emulsion and TQ nanoemulsion in rats fed with a diet rich of high fat-cholesterol for 6 months. The results were very interesting as TQRFNE ameliorated behavioral changes, lipid peroxidation and soluble $A \beta$ levels. Furthermore, there were an improved radical scavenging activity and increased antioxidants genes expression levels in the brain cortex and in the hippocampus. These findings offer a further evidence that 
the presence of different compounds in the preparation (e.g., camphor, carvone, limonene, and thymol) may increase the effect of TQ alone. Moreover, the nanoemulsion may enhance the oral bioavailability and brain delivery.

Overall these results strongly suggest that NS, and its constituent TQ, show interesting proprieties mainly attributable to the balancing of the oxidative processes, whose overt activation represents a pivotal basis of neuroinfiammation and neurodegeneration.

According to the CAMARADES analysis (Table 3), although all the data obtained a good score $(>5)$, we underline a severe gap in all the studies, due to the lacks of details for the allocation concealment. The Al-Majed's study (Al-Majed et al., 2006) presented the highest score (8/10) and it is the only research performed with a blinded assessment of outcome. In four studies the experimental protocols were drawn by combining the biochemical and histological evaluations. Other studies, focused mainly on the memory/behavioral tests such as the passive avoidance test and the classical MWM. Thus, not all the adopted criteria are always applicable.

\section{FUTURE RESEARCH NEEDS AND TRANSLATIONAL PERSPECTIVES IN CLINICAL PRACTICE}

Although experimental studies on the protective role of NS/TQ and other nutraceuticals in $\mathrm{AD}$ report interesting findings, further investigations are needed in order to understand the molecular basis underlying the neuroinflammation and the neurodegenerative processes involved in AD's pathogenesis. An interesting issue concerns the mechanisms responsible for the prolonged activation of the classically activated proinflammatory microglia phenotype 1 (M1) as well as the transition between the M1 phenotype and the alternatively activated phenotypes 2 (M2) which promotes the tissue repairing through the action of anti-inflammatory cytokines (e.g., IL-10, IL-4, IL-3) (Wang et al., 2015). Since a recent study addressed the topic investigating on the flavonoid rutin (Bispo da Silva et al., 2017), it could be interesting to investigate the potential role of NS/TQ in different phases of microglia activation/transaction.

Further research are also needed in order to identify specific cellular and molecular targets. As a consequence, starting from this knowledge, it would be useful to design clinical trials by using several combinations of different compounds, with the scope of engineering an effective translational perspective into clinical practice. Interestingly, the combination of different antioxidants has already been tested successfully and it has been proved that there is a real association between the synergism of combined antioxidants. For instance, Zandi et al. (2004) demonstrated that despite the combination of vitamins $\mathrm{E}, \mathrm{B}$, and $\mathrm{C}$ supplements significantly reduced the risk of $\mathrm{AD}$, the intake of antioxidant vitamins used alone did not reduce the risk of $\mathrm{AD}$ prevalence.

Another strategy of treatment could involve the association between an antioxidant (e.g., NS/TQ) and an agent of targeted therapy. For instance, Kitazawa et al. (2011) blocked in 3xTg$\mathrm{AD}$ mice the IL-1 signaling and demonstrated a reduced neuroinflammation (e.g., nuclear factor kappa-light-chainenhancer of activated B cells, NF-kB, activity), A $\beta$ deposition and tau pathology. Moreover, other investigations have been conducted by blocking TNF signaling with TNF- $\alpha$ monoclonal antibody Infliximab (Shi et al., 2011) or in TNF knock-down

TABLE 3 | The quality of evidence obtained by using a modified CAMARADES (Macleod et al., 2004).

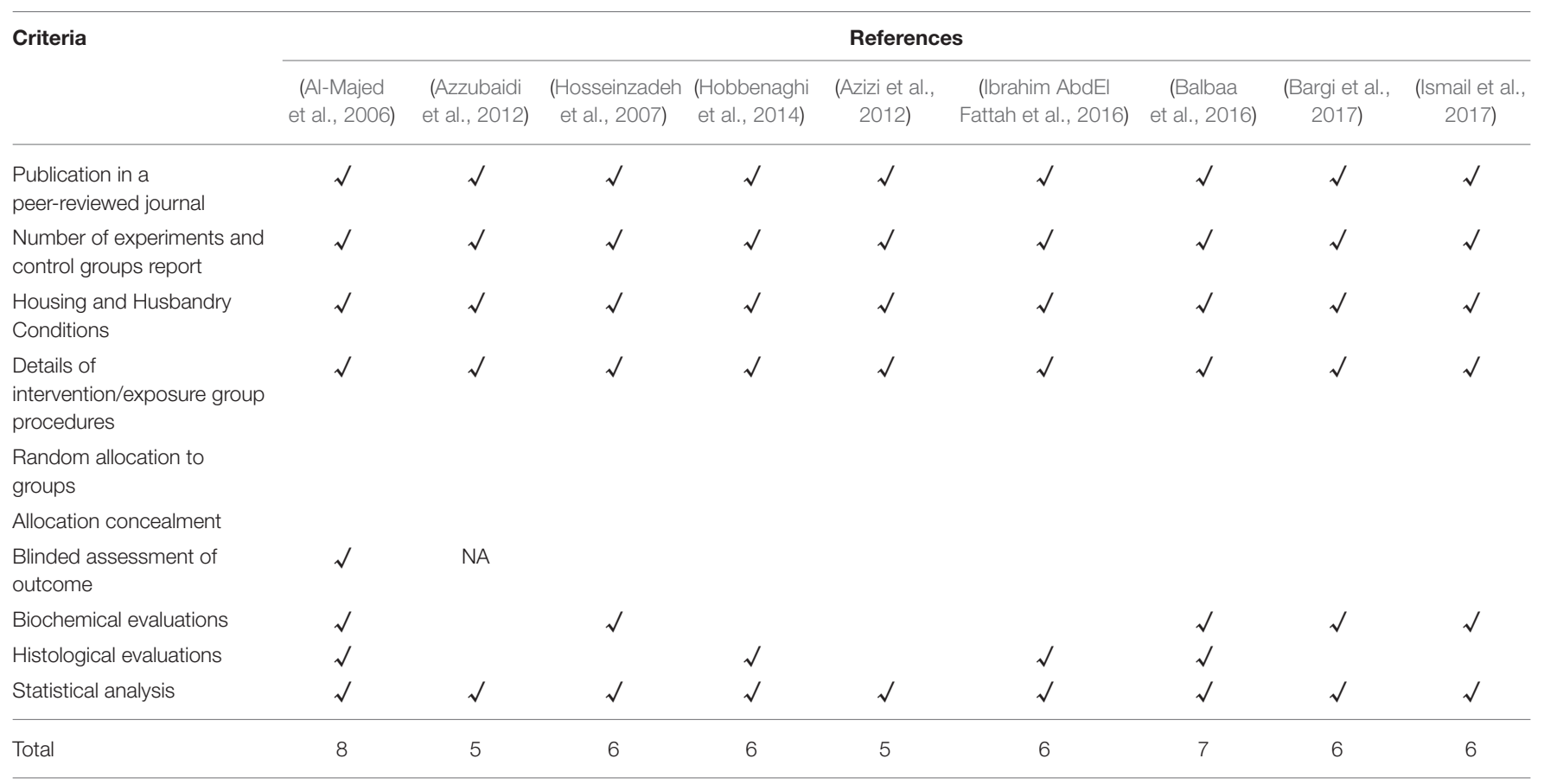

$\sqrt{ }$, the criterion is satisfied; NA, not applicable. 
mice, although the results were mixed (Montgomery et al., 2011). As alternative target for the treatment of AD's syndrome an interesting paper by Ettchetto et al. showed promising findings on the use of dexibuprofen in a mouse model of familial AD, by reducing the neuroinflammatory response, the levels of amyloid plaques and neurofibrillary tangles, while enhancing the memory (Ettcheto et al., 2017).

The preclinical research in this field has the difficult task of offering effective therapeutic opportunities to the clinical investigation whereas, to date, the role of nutraceuticals in $\mathrm{AD}$ prevention and treatment is of a limited clinical importance. For example, the British Association for Psychopharmacology stated that further evidence is needed to recommend the use of vitamin $\mathrm{E}$ and nutritional supplements for this purpose ( $\mathrm{O}^{\prime} \mathrm{Brien}$ et al., 2017). More focused preclinical investigation on the role of nutraceuticals in neurodegenerative diseases could bypass these difficulties and thus could provide the evidence requested by the scientific community.

\section{CONCLUSION}

To date, there is no drug identifiable as a gold standard in the AD's prevention or treatment. To cover this gap, many neuroprotective agents including nutraceuticals have been evaluated for their potential benefits. This review suggests that NS/TQ could have a significant role for preventing and retarding the progression of $\mathrm{AD}$. The promising results of in vitro and in vivo preclinical investigations, and the safety

\section{REFERENCES}

Abdel-Fattah, A. M., Matsumoto, K., and Watanabe, H. (2000). Antinociceptive effects of Nigella sativa oil and its major component, thymoquinone, in mice. Eur. J. Pharmacol. 400, 89-97. doi: 10.1016/S0014-2999(00)00340-X

Agostinho, P., Cunha, R. A., and Oliveira, C. (2010). Neuroinflammation, oxidative stress and the pathogenesis of Alzheimer's disease. Curr. Pharm. Des. 16, 2766-2778. doi: 10.2174/138161210793176572

Aisen, P. S., Schneider, L. S., Sano, M., Diaz-Arrastia, R., van Dyck, C. H., Weiner, M. F., et al. (2008). High-dose B vitamin supplementation and cognitive decline in Alzheimer disease: a randomized controlled trial. JAMA 300, 1774-1783. doi: 10.1001/jama.300.15.1774

Akram Khan, M., and Afzal, M. (2016). Chemical composition of Nigella sativa Linn: part 2 recent advances. Inflammopharmacology 24, 67-79. doi: 10.1007/s10787-016-0262-7

Alhebshi, A. H., Gotoh, M., and Suzuki, I. (2013). Thymoquinone protects cultured rat primary neurons against amyloid beta-induced neurotoxicity. Biochem. Biophys. Res. Commun. 433, 362-367. doi: 10.1016/j.bbrc.2012.11.139

Alhebshi, A. H., Odawara, A., Gotoh, M., and Suzuki, I. (2014). Thymoquinone protects cultured hippocampal and human induced pluripotent stem cellsderived neurons against alpha-synuclein-induced synapse damage. Neurosci. Lett. 570, 126-131. doi: 10.1016/j.neulet.2013.09.049

Aliev, G., Shahida, K., Gan, S. H., Firoz, C., Khan, A., Abuzenadah, A. M., et al. (2014). Alzheimer disease and type 2 diabetes mellitus: the link to tyrosine hydroxylase and probable nutritional strategies. CNS Neurol. Disord. Drug Targets 13, 467-477. doi: 10.2174/18715273113126660153

Al-Majed, A. A., Al-Omar, F. A., and Nagi, M. N. (2006). Neuroprotective effects of thymoquinone against transient forebrain ischemia in the rat hippocampus. Eur. J. Pharmacol. 543, 40-47. doi: 10.1016/j.ejphar.2006.05.046

Andrade, C., and Radhakrishnan, R. (2009). The prevention and treatment of cognitive decline and dementia: an overview of recent research on experimental profile of the compounds, should encourage further preclinical investigations and the translation into clinical practice through the drawing of long-term studies conducted on large population size of AD's patients. In particular, further investigations should be performed to elucidate the effectiveness of NS and its individual components. Indeed, despite TQ showed a better nephro-protective effect, no combinatorial studies on NS and its single compounds, have been performed. Moreover, the pharmacokinetics of these compounds, should be elucidated, since data about the brain's bioavailability and transport across blood-brain barrier, are still inconsistent. More pre-clinical studies will be extremely needed in order to highlight the potential interactions of NS/TQ with other drugs, to elaborate the long-term results and, finally to validate and compare the effectiveness of NS/TQ at different stages of AD.

\section{AUTHOR CONTRIBUTIONS}

MC, SB and AB: written the review protocol, scheduled the inclusion and the exclusion criteria and assessed potential articles for inclusion into the manuscript; $\mathrm{MM}, \mathrm{AV}, \mathrm{AF}$ and $\mathrm{VD}$ : screened and assessed the articles; AF and $\mathrm{AB}$ : independently assessed study quality for the CAMARADES checklist; $A B$, $\mathrm{AC}, \mathrm{CA}$ and AA: assisted all the reviewers; AV, AF, GB, CA: revised the tables. $\mathrm{AA}$ and $\mathrm{GB}$ : revised the English language. All authors partecipate to draft the work, to revise it critically for intellectual contents and approved the final version of manuscript. treatments. Indian J. Psychiatry 51, 12-25. doi: 10.4103/0019-5545. 44900

Azizi, Z., Ebrahimi, S., Saadatfar, E., Kamalinejad, M., and Majlessi, N. (2012). Cognitive-enhancing activity of thymol and carvacrol in two rat models of dementia. Behav. Pharmacol. 23, 241-249. doi: 10.1097/FBP.0b013e3283534301

Azzubaidi, M. S., Saxena, A. K., Talib, N. A., Ahmed, Q. U., and Dogarai, B. B. (2012). Protective effect of treatment with black cumin oil on spatial cognitive functions of rats that suffered global cerebrovascular hypoperfusion. Acta Neurobiol. Exp. 72, 154-165.

Badary, O. A., Taha, R. A., Gamal el-Din, A. M., and Abdel-Wahab, M. H. (2003). Thymoquinone is a potent superoxide anion scavenger. Drug Chem. Toxicol. 26, 87-98. doi: 10.1081/DCT-120020404

Balbaa, M., Abdulmalek, S. A., and Khalil, S. (2017). Oxidative stress and expression of insulin signaling proteins in the brain of diabetic rats: role of Nigella sativa oil and antidiabetic drugs. PLOS ONE 12:e0172429. doi: 10.1371/journal.pone.0172429

Balbaa, M., El-Zeftawy, M., Ghareeb, D., Taha, N., and Mandour, A. W. (2016). Nigella sativa relieves the altered insulin receptor signaling in streptozotocininduced diabetic rats fed with a high-fat diet. Oxid. Med. Cell. Longev. 2016:2492107. doi: 10.1155/2016/2492107

Bamosa, A. O., Kaatabi, H., Lebdaa, F. M., Elq, A. M., and Al-Sultanb, A. (2010). Effect of Nigella sativa seeds on the glycemic control of patients with type 2 diabetes mellitus. Indian J. Physiol. Pharmacol. 54, 344-354.

Bargi, R., Asgharzadeh, F., Beheshti, F., Hosseini, M., Sadeghnia, H. R., and Khazaei, M. (2017). The effects of thymoquinone on hippocampal cytokine level, brain oxidative stress status and memory deficits induced by lipopolysaccharide in rats. Cytokine 96, 173-184. doi: 10.1016/j.cyto.2017.04.015

Bin Sayeed, M. S., Asaduzzaman, M., Morshed, H., Hossain, M. M., Kadir, M. F., and Rahman, M. R. (2013). The effect of Nigella sativa Linn. seed on memory, 
attention and cognition in healthy human volunteers. J. Ethnopharmacol. 148, 780-786. doi: 10.1016/j.jep.2013.05.004

Bin Sayeed, M. S., Shams, T., Fahim Hossain, S., Rahman, M. R., Mostofa, A., Fahim Kadir, M., et al. (2014). Nigella sativa L. seeds modulate mood, anxiety and cognition in healthy adolescent males. J. Ethnopharmacol. 152, 156-162. doi: 10.1016/j.jep.2013.12.050

Bispo da Silva, A., Cerqueira Coelho, P. L., Alves Oliveira Amparo, J., Alves de Almeida Carneiro, M. M., Pereira Borges, J. M., Dos Santos Souza, C., et al. (2017). The flavonoid rutin modulates microglial $/$ macrophage activation to a CD150/CD206 M2 phenotype. Chem. Biol. Interact. 274, 89-99. doi: 10.1016/j.cbi.2017.07.004

Boudiaf, K., Hurtado-Nedelec, M., Belambri, S. A., Marie, J. C., Derradji, Y., Benboubetra, M., et al. (2016). Thymoquinone strongly inhibits fMLF-induced neutrophil functions and exhibits anti-inflammatory properties in vivo. Biochem. Pharmacol. 104, 62-73. doi: 10.1016/j.bcp.2016.01.006

Cascella, M., Bimonte, S., Muzio, M. R., Schiavone, V., and Cuomo, A. (2017a). The efficacy of epigallocatechin-3-gallate (green tea) in the treatment of Alzheimer's disease: an overview of pre-clinical studies and translational perspectives in clinical practice. Infect. Agents Cancer 12:36. doi: 10.1186/s13027-017-0145-6

Cascella, M., Palma, G., Barbieri, A., Bimonte, S., Amruthraj, N. J., Muzio, M. R., et al. (2017b). Role of Nigella sativa and its constituent thymoquinone on chemotherapy-induced nephrotoxicity: evidences from experimental animal studies. Nutrients 9:E625. doi: 10.3390/nu9060625

Cobourne-Duval, M. K., Taka, E., Mendonca, P., Bauer, D., and Soliman, K. F. (2016). The antioxidant effects of thymoquinone in activated BV-2 murine microglial cells. Neurochem. Res. 41, 3227-3238. doi: 10.1007/s11064-016-2047-1

De Domenico, S., and Giudetti, A. M. (2017). Nutraceutical intervention in ageing brain. JGG 65, 79-92.

Doolaanea, A. A., Mansor, N., Mohd Nor, N. H., and Mohamed, F. (2016). Co-encapsulation of Nigella sativa oil and plasmid DNA for enhanced gene therapy of Alzheimer's disease. J. Microencapsul. 33, 114-126. doi: 10.3109/02652048.2015.1134689

Elmaci, I., and Altinoz, M. A. (2016). Thymoquinone: an edible redox-active quinone for the pharmacotherapy of neurodegenerative conditions and glial brain tumors. A short review. Biomed. Pharmacother. 83, 635-640. doi: 10.1016/j.biopha.2016.07.018

Essa, M. M., Mohammed, A., and Guillemin, G. (2016). The Benefits of Natural Products for Neurodegenerative Diseases. Springer.

Ettcheto, M., Sánchez-López, E., Pons, L., Busquets, O., Olloquequi, J., Beas-Zarate, C., et al. (2017). Dexibuprofen prevents neurodegeneration and cognitive decline in APPswe/PS1dE9 through multiple signaling pathways. Redox Biol. 13, 345-352. doi: 10.1016/j.redox.2017.06.003

Gholamnezhad, Z., Havakhah, S., and Boskabady, M. H. (2016). Preclinical and clinical effects of Nigella sativa and its constituent, thymoquinone: a review. $J$ Ethnopharmacol. 190, 372-386. doi: 10.1016/j.jep.2016.06.061

Hayatdavoudi, P., Khajavi Rad, A., Rajaei, Z., and Hadjzadeh, M. A. (2016). Renal injury, nephrolithiasis and Nigella sativa: a mini review. Avicenna J. Phytomed. $6,1-8$.

Hobbenaghi, R., Javanbakht, J., Sadeghzadeh, S. H., Kheradmand, D., Abdi, F. S., Jaberi, M. H., et al. (2014). Neuroprotective effects of Nigella sativa extract on cell death in hippocampal neurons following experimental global cerebral ischemia-reperfusion injury in rats. J. Neurol. Sci. 337, 74-79. doi: 10.1016/j.jns.2013.11.019

Hosseinzadeh, H., Parvardeh, S., Asl, M. N., Sadeghnia, H. R., and Ziaee, T. (2007). Effect of thymoquinone and Nigella sativa seed oil on lipid peroxidation level during global cerebral ischemia-reperfusion injury in rat hippocampus. Phytomedicine 14, 621-627. doi: 10.1016/j.phymed.2006.12.005

Iadecola, C. (2010). The overlap between neurodegenerative and vascular factors in the pathogenesis of dementia. Acta Neuropathol. 120, 287-296. doi: 10.1007/s00401-010-0718-6

Ibrahim AbdEl Fattah, L., Zickri, M. B., Aal, L. A., Heikal, O., and Osama, E. (2016). The effect of thymoquinone, alpha7 receptor agonist and alpha7 receptor allosteric modulator on the cerebral cortex in experimentally induced Alzheimer's disease in relation to MSCs activation. Int. J. Stem Cells 9, 230-238. doi: 10.15283/ijsc16021

Ismail, N., Ismail, M., Azmi, N. H., Bakar, M. F. A., Yida, Z., Stanslas, J., et al. (2017). Beneficial effects of TQRF and TQ nano- and conventional emulsions on memory deficit, lipid peroxidation, total antioxidant status, antioxidants genes expression and soluble $\mathrm{A} \beta$ levels in high fat-cholesterol diet-induced rats. Chem. Biol. Interact. 275, 61-7359. doi: 10.1016/j.cbi.2017.07.014

Ismail, N., Ismail, M., Latiff, L. A., Mazlan, M., and Mariod, A. A. (2008). Black cumin seed (Nigella sativa linn.) oil and its fractions protect against beta amyloid peptide-induced toxicity in primary cerebellar granule neurons. J. Food Lipids 15, 519-533. doi: 10.1111/j.1745-4522.2008.00137.x

Ismail, N., Ismail, M., Mazlan, M., Latiff, L. A., Imam, M. U., Iqbal, S., et al. (2013). Thymoquinone prevents beta-amyloid neurotoxicity in primary cultured cerebellar granule neurons. Cell. Mol. Neurobiol. 33, 1159-1169. doi: 10.1007/s10571-013-9982-z

Jaarin, K., Foong, W. D., Yeoh, M. H., Kamarul, Z. Y., Qodriyah, H. M., Azman, A., et al. (2015). Mechanisms of the antihypertensive effects of Nigella sativa oil in L-NAME-induced hypertensive rats. Clinics 70, 751-757. doi: 10.6061/clinics/2015(11)07

Javidi, S., Razavi, B. M., and Hosseinzadeh, H. (2016). A review of Neuropharmacology effects of Nigella sativa and its main component, thymoquinone. Phytother. Res. 30, 1219-1229. doi: 10.1002/ptr.5634

Jukic, M., Politeo, O., Maksimovic, M., Milos, M., and Milos, M. (2007). in vitro acetylcholinesterase inhibitory properties of thymol, carvacrol and their derivatives thymoquinone and thymohydroquinone. Phytother. Res. 21, 259-261. doi: 10.1002/ptr.2063

Kennedy, K., Tucci, M. A., and Benghuzzi, H. A. (2014). Comparison of potential preventive therapeutic agents green tea, thymoquinone, and dilinoleoylphosphatidylcholine on human neuroblastoma cells. Biomed. Sci. Instrum. 50, 132-139.

Keyhanmanesh, R., Boskabady, M. H., Eslamizadeh, M. J., Khamneh, S., and Ebrahimi, M. A. (2010). The effect of thymoquinone, the main constituent of Nigella sativa on tracheal responsiveness and white blood cell count in lung lavage of sensitized guinea pigs. Planta Med. 76, 218-222. doi: 10.1055/s-0029-1186054

Khan, A., Vaibhav, K., Javed, H., Khan, M. M., Tabassum, R., Ahmed, M. E., et al. (2012). Attenuation of Abeta-induced neurotoxicity by thymoquinone via inhibition of mitochondrial dysfunction and oxidative stress. Mol. Cell. Biochem. 369, 55-65. doi: 10.1007/s11010-012-1368-x

Kitazawa, M., Cheng, D., Tsukamoto, M. R., Koike, M. A., Wes, P. D., Vasilevko, V., et al. (2011). Blocking IL-1 signaling rescues cognition, attenuates tau pathology, and restores neuronal $\beta$-catenin pathway function in an Alzheimer's disease model. J. Immunol. 187, 6539-6549. doi: 10.4049/jimmunol. 1100620

Kundu, J., Chun, K.-S., Aruoma, O. I., and Kundu, J. K. (2014). Mechanistic perspectives on cancer chemoprevention/chemotherapeutic effects of thymoquinone. Mutat. Res. 768, 22-34. doi: 10.1016/j.mrfmmm.2014.05.003

Laver, K., Dyer, S., Whitehead, C., Clemson, L., and Crotty, M. (2016). Interventions to delay functional decline in people with dementia: a systematic review of systematic reviews. BMJ Open 6:e010767. doi: 10.1136/bmjopen-2015-010767

Lei, Y., Chen, J., Zhang, W., Fu, W., Wu, G., Wei, H., et al. (2012). In vivo investigation on the potential of galangin, kaempferol and myricetin for protection of D-galactose-induced cognitive impairment. Food Chem. 135, 2702-2707. doi: 10.1016/j.foodchem.2012.07.043

Liberati, A., Altman, D. G., Tetzlaff, J., Mulrow, C., Gøtzsche, P. C., Ioannidis, J. P. A., et al. (2009). The PRISMA statement for reporting systematic reviews and meta-analyses of studies that evaluate healthcare interventions: explanation and elaboration. BMJ 339:b270. doi: 10.1136/bmj.b2700

Lim, G. P., Chu, T., Yang, F., Beech, W., Frautschy, S. A., and Cole, G. M. (2001). The curry spice curcumin reduces oxidative damage and amyloid pathology in an Alzheimer transgenic mouse. J. Neurosci. 21, 8370-8377.

Liu, X., Abd El-Aty, A. M., and Shim, J. H. (2011). Various extraction and analytical techniques for isolation and identification of secondary metabolites from Nigella sativa seeds. Mini Rev. Med. Chem. 11, 947-955. doi: 10.2174/138955711797068472

Macleod, M. R., O’Collins, T., Howells, D. W., and Donnan, G. A. (2004). Pooling of animal experimental data reveals influence of study design and publication bias. Stroke 35, 1203-1208. doi: 10.1161/01.STR.0000125719.25853.20

Mecocci, P., Tinarelli, C., Schulz, R. J., and Polidori, M. C. (2014). Nutraceuticals in cognitive impairment and Alzheimer's disease. Front. Pharmacol. 5:147. doi: 10.3389/fphar.2014.00147 
Montgomery, S. L., Mastrangelo, M. A., Habib, D., Narrow, W. C., Knowlden, S. A., Wright, T. W., et al. (2011). Ablation of TNF-RI/RII expression in Alzheimer's disease mice leads to an unexpected enhancement of pathology: implications for chronic pan-TNF- $\alpha$ suppressive therapeutic strategies in the brain. Am. J. Pathol. 179, 2053-2070. doi: 10.1016/j.ajpath.2011.07.001

Mosher, K. I., and Wyss-Coray, T. (2014). Microglial dysfunction in brain aging and Alzheimer's disease. J. Biochemical. Pharmacol. 88, 594-604. doi: 10.1016/j.bcp.2014.01.008

O’Brien, J. T., Holmes, C., Jones, M., Jones, R., Livingston, G., McKeith, I., et al. (2017). Clinical practice with anti-dementia drugs: a revised (third) consensus statement from the British Association for Psychopharmacology. J. Psychopharmacol. 31, 147-169. doi: 10.1177/0269881116680924

Poulose, S. M., Miller, M. G., Scott, T., and Shukitt-Hale, B. (2017). Nutritional factors affecting adult neurogenesis and cognitive function. Adv. Nutr. 8, 804-811. doi: 10.3945/an.117.016261

Ragheb, A., Attia, A., Eldin, W., Elbarbry, F., Gazarin, S., and Shoker, A. (2009). The protective effect of thymoquinone, an anti-oxidant and anti-inflammatory agent, against renal injury: a review. Saudi J. Kidney Dis. Transpl. 20, 741-752.

Sahak, M. K., Kabir, N., Abbas, G., Draman, S., Hashim, N. H., and Hasan Adli, D. S. (2016). The role of Nigella sativa and its active constituents in learning and memory. Evid Based Complement Alternat. Med. 2016:6075679. doi: $10.1155 / 2016 / 6075679$

Sahak, M. K., Mohamed, A. M., Hashim, N. H., and Hasan Adli, D. S. (2013). Nigella sativa oil enhances the spatial working memory performance of rats on a radial arm maze. Evid Based Complement. Alternat. Med. 2013:180598. doi: 10.1155/2013/180598

Schültke, E., Kendall, E., Kamencic, H., Ghong, Z., Griebel, R. W., and Juurlink, B. H. (2003). Quercetin promotes functional recovery following acute spinal cord injury. J. Neurotrauma 20, 583-591. doi: 10.1089/08977150376 7168500

Shi, J.-Q., Shen, W., Chen, J., Wang, B.-R., Zhong, L.-L., Zhu, Y.-W., et al. (2011). Anti-TNF- $\alpha$ reduces amyloid plaques and tau phosphorylation and induces
CD11c-positive dendritic-like cell in the APP/PS1 transgenic mouse brains. Brain Res. 1368, 239-247. doi: 10.1016/j.brainres.2010.10.053

Wang, W. Y., Tan, M. S., Yu, J. T., and Tan, L. (2015). Role of pro-inflammatory cytokines released from microglia in Alzheimer's disease. Ann. Transl. Med. 3:136. doi: 10.3978/j.issn.2305-5839.2015.03.49

Yildiz, F., Coban, S., Terzi, A., Ates, M., Aksoy, N., Cakir, H., et al. (2008). Nigella sativa relieves the deleterious effects of ischemia reperfusion injury on liver. World J. Gastroenterol. 14, 5204-5209. doi: 10.3748/wjg.14.5204

Yuan, Q., Wang, C. W., Shi, J., and Lin, Z. X. (2017). Effects of Ginkgo biloba on dementia: an overview of systematic reviews. J. Ethnopharmacol. 195, 1-9. doi: 10.1016/j.jep.2016.12.005

Zandi, P. P., Anthony, J. C., Khachaturian, A. S., Stone, S. V., Gustafson, D., Tschanz, J. T., et al. (2004). Reduced risk of Alzheimer disease in users of antioxidant vitamin supplements: the cache county study. Arch. Neurol. 61, 82-88. doi: 10.1001/archneur.61.1.82

Zhao, H. F., Li, N., Wang, Q., Cheng, X. J., Li, X. M., and Liu, T. T. (2015). Resveratrol decreases the insoluble $A \beta 1-42$ level in hippocampus and protects the integrity of the blood-brain barrier in AD rats. Neuroscience 310, 641-649. doi: 10.1016/j.neuroscience.2015.10.006

Conflict of Interest Statement: The authors declare that the research was conducted in the absence of any commercial or financial relationships that could be construed as a potential conflict of interest.

Copyright (c) 2018 Cascella, Bimonte, Barbieri, Del Vecchio, Muzio, Vitale, Benincasa, Ferriello, Azzariti, Arra and Cuomo. This is an open-access article distributed under the terms of the Creative Commons Attribution License (CC $B Y)$. The use, distribution or reproduction in other forums is permitted, provided the original author(s) and the copyright owner are credited and that the original publication in this journal is cited, in accordance with accepted academic practice. No use, distribution or reproduction is permitted which does not comply with these terms. 